\title{
An Eigenvalue Problem for a Fermi System and Lie Algebras
}

\author{
Willi-Hans Steeb ${ }^{\dagger}$ and Yorick Hardy* \\ $\dagger$ International School for Scientific Computing, \\ University of Johannesburg, Auckland Park 2006, South Africa, \\ e-mail: steebwilli@gmail.com \\ * Department of Mathematical Sciences, \\ University of South Africa, Pretoria, South Africa, \\ e-mail: hardyy@unisa.ac.za
}

\begin{abstract}
We study a Fermi Hamilton operator $\hat{K}$ which does not commute with the number operator $\hat{N}$. The eigenvalue problem and the Schrödinger equation is solved. Entanglement is also discussed. Furthermore the Lie algebra generated by the two terms of the Hamilton operator is derived and the Lie algebra generated by the Hamilton operator and the number operator is also classified.
\end{abstract}

\section{Introduction}

In quantum theory Hamilton operators with Fermi-interactions have a long history [1, 2, 3, 4, 5, 6, 7, 8, 9. Entanglement for Hamilton operators with Fermiinteractions has been studied by many authors [10, 11, 12, 13]. Let $c_{j}^{\dagger}, c_{j}(j=$ $1, \ldots, n)$ be (spin-less) Fermi creation and annihilation operators, i.e.

$$
\left[c_{j}^{\dagger}, c_{k}\right]_{+}=\delta_{j k} I, \quad\left[c_{j}, c_{k}\right]_{+}=0, \quad\left[c_{j}^{\dagger}, c_{k}^{\dagger}\right]_{+}=0
$$

where $[,]_{+}$denotes the anticommutator and $I$ is the identity operator. Let $|0\rangle$ be the vacuum state. Then $c_{j}|0\rangle=0$ and $\langle 0 \mid 0\rangle=1$. Here we study the self-adjoint Hamilton operator

$$
\hat{K}=\frac{\hat{H}}{\hbar \omega}=c_{n}^{\dagger} c_{n-1}^{\dagger} \cdots c_{2}^{\dagger} c_{1}^{\dagger}+c_{1} c_{2} \cdots c_{n-1} c_{n} .
$$

The number operator $\hat{N}$ is given by

$$
\hat{N}=\sum_{j=1}^{n} c_{j}^{\dagger} c_{j}
$$


Obviously $[\hat{K}, \hat{N}] \neq 0$. We find the matrix representation of $\hat{K}$ and its eigenvalues and eigenvectors. We utilize the faithful matrix representation [6, 7, 8] for Fermi operators

$$
\begin{gathered}
c_{k}^{\dagger}=\overbrace{\sigma_{3} \otimes \cdots \otimes \sigma_{3} \otimes\left(\frac{1}{2} \sigma_{+}\right) \otimes I_{2} \otimes \cdots \otimes I_{2}}^{n \text {-times }} \\
c_{k}=\sigma_{3} \otimes \cdots \otimes \sigma_{3} \otimes\left(\frac{1}{2} \sigma_{-}\right) \otimes I_{2} \otimes \cdots \otimes I_{2} \\
k \text {-th place }
\end{gathered}
$$

where $I_{2}$ is the $2 \times 2$ identity matrix and $\sigma_{1}, \sigma_{2}, \sigma_{3}$ are the Pauli spin matrices

$$
\sigma_{1}=\left(\begin{array}{ll}
0 & 1 \\
1 & 0
\end{array}\right), \quad \sigma_{2}=\left(\begin{array}{cc}
0 & -i \\
i & 0
\end{array}\right), \quad \sigma_{3}=\left(\begin{array}{cc}
1 & 0 \\
0 & -1
\end{array}\right)
$$

with

$$
\sigma_{+}=\sigma_{1}+i \sigma_{2}=\left(\begin{array}{cc}
0 & 2 \\
0 & 0
\end{array}\right), \quad \sigma_{-}=\sigma_{1}-i \sigma_{2}=\left(\begin{array}{cc}
0 & 0 \\
2 & 0
\end{array}\right)
$$

We also calculate the unitary matrix $U(t)=\exp (-i \hat{H} t / \hbar)$ to solve the Schrödinger and Heisenberg equation of motion. Entangled and unentangled states can be found. As entanglement measure for the eigenvectors of Hamilton operator $\hat{K}$ we utilize the entanglement measure introduced by Wong and Christensen [14].

We also study the Lie algebra generated by $c_{n}^{\dagger} c_{n-1}^{\dagger} \cdots c_{2}^{\dagger} c_{1}^{\dagger}$ and $c_{1} c_{2} \cdots c_{n-1} c_{n}$ and the Lie algebra generated by the Hamilton operator $\hat{K}$ and the number operator $\hat{N}$.

\section{Eigenvalue Problem for the Cases $n=1$ and $n=2$}

For the case $n=1$ we have the Hamilton operator

$$
\hat{K}=\frac{\hat{H}}{\hbar \omega}=c^{\dagger}+c=\left(\begin{array}{ll}
0 & 1 \\
1 & 0
\end{array}\right)=\sigma_{1}
$$

where the operators $c^{\dagger}, c$ and $c^{\dagger} c$ are given by the $2 \times 2$ matrices

$$
c^{\dagger}=\left(\begin{array}{ll}
0 & 1 \\
0 & 0
\end{array}\right), \quad c=\left(\begin{array}{ll}
0 & 0 \\
1 & 0
\end{array}\right), \quad \hat{N}=c^{\dagger} c=\left(\begin{array}{ll}
1 & 0 \\
0 & 0
\end{array}\right)
$$


and the basis is given by

$$
c^{\dagger}|0\rangle=\left(\begin{array}{l}
1 \\
0
\end{array}\right), \quad|0\rangle=\left(\begin{array}{l}
0 \\
1
\end{array}\right) .
$$

Thus the Hamilton operator $\hat{H}$ acts in the Hilbert space $\mathbb{C}^{2}$. Obviously the eigenvalues of $\hat{K}$ are +1 and -1 with the corresponding normalized eigenvectors (Hadamard basis)

$$
\frac{1}{\sqrt{2}}\left(\begin{array}{l}
1 \\
1
\end{array}\right), \quad \frac{1}{\sqrt{2}}\left(\begin{array}{c}
1 \\
-1
\end{array}\right)
$$

For the unitary operator $U(t)=\exp (-i \hat{H} t / \hbar)$ we obtain

$$
U(t)=\exp (-i \hat{H} t / \hbar)=\left(\begin{array}{cc}
\cos (\omega t) & -i \sin (\omega t) \\
-i \sin (\omega t) & \cos (\omega t)
\end{array}\right) .
$$

Consider now the case $n=2$. The ordering of the four dimensional basis is $c_{2}^{\dagger} c_{1}^{\dagger}|0\rangle$, $c_{2}^{\dagger}|0\rangle, c_{1}^{\dagger}|0\rangle,|0\rangle$. Utilizing the matrix representation given above we have

$$
c_{1}=\frac{1}{2} \sigma_{-} \otimes I_{2}, \quad c_{2}=\sigma_{3} \otimes \frac{1}{2} \sigma_{-} .
$$

Thus we obtain the matrix representation

$$
c_{1} c_{2}=\left(\begin{array}{cccc}
0 & 0 & 0 & 0 \\
0 & 0 & 0 & 0 \\
0 & 0 & 0 & 0 \\
1 & 0 & 0 & 0
\end{array}\right), \quad c_{2}^{\dagger} c_{1}^{\dagger}=\left(\begin{array}{cccc}
0 & 0 & 0 & 1 \\
0 & 0 & 0 & 0 \\
0 & 0 & 0 & 0 \\
0 & 0 & 0 & 0
\end{array}\right)
$$

Consequently

$$
\hat{K}=c_{2}^{\dagger} c_{1}^{\dagger}+c_{1} c_{2}=\left(\begin{array}{cccc}
0 & 0 & 0 & 1 \\
0 & 0 & 0 & 0 \\
0 & 0 & 0 & 0 \\
1 & 0 & 0 & 0
\end{array}\right), \quad\left[c_{2}^{\dagger} c_{1}^{\dagger}, c_{1} c_{2}\right]=\left(\begin{array}{cccc}
1 & 0 & 0 & 0 \\
0 & 0 & 0 & 0 \\
0 & 0 & 0 & 0 \\
0 & 0 & 0 & -1
\end{array}\right) .
$$

The four eigenvalues of $\hat{K}$ are $-1,1,0$ (twice) with the corresponding bases for the eigenspaces

$$
\left\{\frac{1}{\sqrt{2}}\left(\begin{array}{c}
1 \\
0 \\
0 \\
-1
\end{array}\right)\right\}, \quad\left\{\frac{1}{\sqrt{2}}\left(\begin{array}{l}
1 \\
0 \\
0 \\
1
\end{array}\right)\right\}, \quad\left\{\left(\begin{array}{l}
0 \\
1 \\
0 \\
0
\end{array}\right),\left(\begin{array}{l}
0 \\
0 \\
1 \\
0
\end{array}\right)\right\} .
$$


The first two eigenspaces have Bell states as basis and are fully entangled (except for the zero vector). The last eigenspace consists of unentangled and entangled vectors. For the number operator $\hat{N}$ we find

$$
\hat{N}=c_{1}^{\dagger} c_{1}+c_{2}^{\dagger} c_{2}=\left(\begin{array}{cccc}
2 & 0 & 0 & 0 \\
0 & 1 & 0 & 0 \\
0 & 0 & 1 & 0 \\
0 & 0 & 0 & 0
\end{array}\right)
$$

with eigenvalues 2, 1 (twice) and 0 and

$$
\left\{\left(\begin{array}{l}
1 \\
0 \\
0 \\
0
\end{array}\right)\right\}, \quad\left\{\left(\begin{array}{l}
0 \\
1 \\
0 \\
0
\end{array}\right),\left(\begin{array}{l}
0 \\
0 \\
1 \\
0
\end{array}\right)\right\}, \quad\left\{\left(\begin{array}{l}
0 \\
0 \\
0 \\
1
\end{array}\right)\right\}
$$

as basis for the respective eigenspaces. For the unitary operator $U(t)=\exp (-i \hat{H} t / \hbar)$ we obtain

$$
U(t)=\exp (-i \hat{H} t / \hbar)=\left(\begin{array}{cccc}
\cos (\omega t) & 0 & 0 & -i \sin (\omega t) \\
0 & 1 & 0 & 0 \\
0 & 0 & 1 & 0 \\
-i \sin (\omega t) & 0 & 0 & \cos (\omega t)
\end{array}\right)
$$

\section{General Case}

For arbitrary $n$ and $n \geq 2$ the Hamilton operator $\hat{K}$ is given by the $2^{n} \times 2^{n}$ symmetric matrix over $\mathbb{R}$ with 1 at the entries $\left(1,2^{n}\right)$ and $\left(2^{n}, 1\right)$ and otherwise 0 , i.e.

$$
\hat{K}=\left(\begin{array}{ll}
0 & 1 \\
0 & 0
\end{array}\right) \otimes \cdots \otimes\left(\begin{array}{ll}
0 & 1 \\
0 & 0
\end{array}\right)+\left(\begin{array}{ll}
0 & 0 \\
1 & 0
\end{array}\right) \otimes \cdots \otimes\left(\begin{array}{ll}
0 & 0 \\
1 & 0
\end{array}\right)
$$

The commutator $\left[c_{n}^{\dagger} c_{n-1}^{\dagger} \cdots c_{2}^{\dagger} c_{1}^{\dagger}, c_{1} c_{2} \cdots c_{n-1} c_{n}\right]$ admits the matrix representation

$$
\left(\begin{array}{ll}
1 & 0 \\
0 & 0
\end{array}\right) \otimes \cdots \otimes\left(\begin{array}{ll}
1 & 0 \\
0 & 0
\end{array}\right)-\left(\begin{array}{ll}
0 & 0 \\
0 & 1
\end{array}\right) \otimes \cdots \otimes\left(\begin{array}{ll}
0 & 0 \\
0 & 1
\end{array}\right) .
$$

This means we have a $2^{n} \times 2^{n}$ diagonal matrix with 1 at the entry $(1,1)$ and -1 at the entry $\left(2^{n}, 2^{n}\right)$ and otherwise 0 . The eigenvalues of $\hat{K}$ are given by $1,-1$ and 0 
$\left(2^{n}-2\right.$ times). The corresponding bases for the eigenspaces are

$$
\left\{\frac{1}{\sqrt{2}}\left(\begin{array}{c}
1 \\
0 \\
\vdots \\
0 \\
1
\end{array}\right)\right\}, \quad\left\{\frac{1}{\sqrt{2}}\left(\begin{array}{c}
1 \\
0 \\
\vdots \\
0 \\
-1
\end{array}\right)\right\}, \quad\left\{\left(\begin{array}{c}
0 \\
1 \\
\vdots \\
0 \\
0
\end{array}\right), \ldots,\left(\begin{array}{c}
0 \\
0 \\
\vdots \\
1 \\
0
\end{array}\right)\right\}
$$

The first two eigenspaces consist of entangled vectors (except for the zero vector). The other $2^{n}-2$ dimensional eigenspace includes entangled and unentangled vectors. For the unitary operator $U(t)=\exp (-i \hat{H} t / \hbar)$ we obtain

$$
\exp (-i \hat{H} t / \hbar)=\left(\begin{array}{ccccc}
\cos (\omega t) & 0 & \ldots & 0 & -i \sin (\omega t) \\
0 & 1 & \ldots & 0 & 0 \\
\vdots & \vdots & \ddots & \vdots & \vdots \\
0 & 0 & \ldots & 1 & 0 \\
-i \sin (\omega t) & 0 & \ldots & 0 & \cos (\omega t)
\end{array}\right)
$$

\section{Lie Algebras}

We are looking first at the Lie algebra generated by the two operators

$$
c_{n}^{\dagger} c_{n-1}^{\dagger} \cdots c_{2}^{\dagger} c_{1}^{\dagger}, \quad c_{1} c_{2} \cdots c_{n-1} c_{n} .
$$

Consider first the case $n=1$. Since $\left[c^{\dagger}, c\right]=2 c^{\dagger} c-I$ and

$$
\left[c^{\dagger}, 2 c^{\dagger} c-I\right]=-2 c^{\dagger}, \quad\left[c, 2 c^{\dagger} c-I\right]=2 c
$$

we find a three-dimensional simple Lie algebra with the basis

$$
c^{\dagger}, \quad c, \quad c^{\dagger} c-\frac{I}{2} .
$$

Thus we have a basis of the simple Lie algebra $s \ell(2, \mathbb{R})$. The matrix representation is

$$
c^{\dagger}=\left(\begin{array}{ll}
0 & 1 \\
0 & 0
\end{array}\right), \quad c=\left(\begin{array}{cc}
0 & 0 \\
1 & 0
\end{array}\right), \quad c^{\dagger} c-\frac{I}{2}=\frac{1}{2}\left(\begin{array}{cc}
1 & 0 \\
0 & -1
\end{array}\right) .
$$

Consider now the case with $n=2$ and the Lie algebra generated by $c_{2}^{\dagger} c_{1}^{\dagger}$ and $c_{1} c_{2}$. We have

$$
\left[c_{2}^{\dagger} c_{1}^{\dagger}, c_{1} c_{2}\right]=c_{1}^{\dagger} c_{1}+c_{2}^{\dagger} c_{2}-I
$$


Next we obtain the commutators

$$
\left[c_{2}^{\dagger} c_{1}^{\dagger}, c_{1}^{\dagger} c_{1}+c_{2}^{\dagger} c_{2}-I\right]=2 c_{1}^{\dagger} c_{2}^{\dagger}, \quad\left[c_{1} c_{2}, c_{1}^{\dagger} c_{1}+c_{2}^{\dagger} c_{2}-I\right]=2 c_{1} c_{2} .
$$

Thus we have a simple three-dimensional Lie algebra with the basis $c_{2}^{\dagger} c_{1}^{\dagger}, c_{1} c_{2}$, $c_{1}^{\dagger} c_{1}+c_{2}^{\dagger} c_{2}-I$. The Lie algebra is isomorphic to $s \ell(2, \mathbb{R})$. The matrix representation given by the diagonal matrix

$$
c_{1}^{\dagger} c_{1}+c_{2}^{\dagger} c_{2}-I=\left(\begin{array}{cccc}
1 & 0 & 0 & 0 \\
0 & 0 & 0 & 0 \\
0 & 0 & 0 & 0 \\
0 & 0 & 0 & -1
\end{array}\right)
$$

and

$$
c_{1} c_{2}=\left(\begin{array}{cccc}
0 & 0 & 0 & 0 \\
0 & 0 & 0 & 0 \\
0 & 0 & 0 & 0 \\
1 & 0 & 0 & 0
\end{array}\right), \quad c_{2}^{\dagger} c_{1}^{\dagger}=\left(\begin{array}{cccc}
0 & 0 & 0 & 1 \\
0 & 0 & 0 & 0 \\
0 & 0 & 0 & 0 \\
0 & 0 & 0 & 0
\end{array}\right)
$$

Consider now the case $n=3$ and the Lie algebra generated by the operators $c_{3}^{\dagger} c_{2}^{\dagger} c_{1}^{\dagger}$ and $c_{1} c_{2} c_{3}$. We obtain the commutator $\left[c_{3}^{\dagger} c_{2}^{\dagger} c_{1}^{\dagger}, c_{1} c_{2} c_{3}\right]=2 c_{3}^{\dagger} c_{2}^{\dagger} c_{1}^{\dagger} c_{1} c_{2} c_{3}-c_{2}^{\dagger} c_{1}^{\dagger} c_{1} c_{2}-c_{3}^{\dagger} c_{2}^{\dagger} c_{2} c_{3}-c_{3}^{\dagger} c_{1}^{\dagger} c_{1} c_{3}+c_{1}^{\dagger} c_{1}+c_{2}^{\dagger} c_{2}+c_{3}^{\dagger} c_{3}-I$.

Next we find

$$
\begin{aligned}
& {\left[c_{1} c_{2} c_{3},\left[c_{3}^{\dagger} c_{2}^{\dagger} c_{1}^{\dagger}, c_{1} c_{2} c_{3}\right]\right]=2 c_{1} c_{2} c_{3}} \\
& {\left[c_{3}^{\dagger} c_{2}^{\dagger} c_{1}^{\dagger},\left[c_{3}^{\dagger} c_{2}^{\dagger} c_{1}^{\dagger}, c_{1} c_{2} c_{3}\right]\right]=-2 c_{3}^{\dagger} c_{2}^{\dagger} c_{1}^{\dagger} .}
\end{aligned}
$$

Thus the three operators $c_{1} c_{2} c_{3}, c_{3}^{\dagger} c_{2}^{\dagger} c_{1}^{\dagger},\left[c_{3}^{\dagger} c_{2}^{\dagger} c_{1}^{\dagger}, c_{1} c_{2} c_{3}\right]$ form a basis of a threedimensional Lie algebra which is isomorphic to $s \ell(2, \mathbb{R})$. The matrix representation of $\left[c_{3}^{\dagger} c_{2}^{\dagger} c_{1}^{\dagger}, c_{1} c_{2} c_{3}\right]$ is given by

$$
\left(\begin{array}{ll}
1 & 0 \\
0 & 0
\end{array}\right) \otimes\left(\begin{array}{ll}
1 & 0 \\
0 & 0
\end{array}\right) \otimes\left(\begin{array}{ll}
1 & 0 \\
0 & 0
\end{array}\right)-\left(\begin{array}{ll}
0 & 0 \\
0 & 1
\end{array}\right) \otimes\left(\begin{array}{ll}
0 & 0 \\
0 & 1
\end{array}\right) \otimes\left(\begin{array}{ll}
0 & 0 \\
0 & 1
\end{array}\right) .
$$

For arbitrary $n$ we have

$$
\begin{aligned}
& {\left[c_{1} \cdots c_{n},\left[c_{n}^{\dagger} \cdots c_{1}^{\dagger}, c_{1} \cdots c_{n}\right]\right]=2 c_{1} \cdots c_{n}} \\
& {\left[c_{n}^{\dagger} \cdots c_{1}^{\dagger},\left[c_{n}^{\dagger} \cdots c_{1}^{\dagger}, c_{1} \cdots c_{n}\right]\right]=-2 c_{n}^{\dagger} \cdots c_{1}^{\dagger} .}
\end{aligned}
$$

Thus for arbitrary $n$ the three operators

$$
c_{n}^{\dagger} c_{n-1}^{\dagger} \cdots c_{2}^{\dagger} c_{1}^{\dagger}, \quad c_{1} c_{2} \cdots c_{n-1} c_{n}, \quad\left[c_{n}^{\dagger} c_{n-1}^{\dagger} \cdots c_{2}^{\dagger} c_{1}^{\dagger}, c_{1} c_{2} \cdots c_{n-1} c_{n}\right]
$$


form a basis of a three dimensional Lie algebra which is isomorphic to $s \ell(2, \mathbb{R})$.

Next we study the Lie algebra generated by the Hamilton operator $\hat{K}$ and the number operator $\hat{N}$. Let $n=1$. We find for the commutators

$[\hat{K}, \hat{N}]=c-c^{\dagger}, \quad\left[\hat{K}, c-c^{\dagger}\right]=4 c^{\dagger} c-2 I=4 \hat{N}-2 I, \quad\left[\hat{N}, c-c^{\dagger}\right]=-c^{\dagger}-c=-\hat{K}$.

Thus we have a four-dimensional non-commutative Lie algebra with a basis given by $\hat{K}, \hat{N}, c-c^{\dagger}, I$. Owing to the operator $I$ the Lie algebra is not semisimple. Utilizing the matrix representation we have

$\hat{K}=\left(\begin{array}{ll}0 & 1 \\ 1 & 0\end{array}\right)=\sigma_{1}, \quad \hat{N}=\left(\begin{array}{ll}1 & 0 \\ 0 & 0\end{array}\right), \quad c-c^{\dagger}=\left(\begin{array}{cc}0 & -1 \\ 1 & 0\end{array}\right)=-i \sigma_{1}, \quad I=\left(\begin{array}{ll}1 & 0 \\ 0 & 1\end{array}\right)$.

For $n=2$ we have the commutators

$$
\begin{aligned}
{[\hat{K}, \hat{N}] } & =2\left(c_{1} c_{2}-c_{2}^{\dagger} c_{1}^{\dagger}\right) \\
{[\hat{K},[\hat{K}, \hat{N}]] } & =4(\hat{N}-I) \\
{[\hat{N},[\hat{K}, \hat{N}]] } & =-4 \hat{K} \\
{[\hat{K},[\hat{K},[\hat{K}, \hat{N}]]] } & =4[\hat{K}, \hat{N}] \\
{[\hat{N},[\hat{K},[\hat{K}, \hat{N}]]] } & =0 \\
{[\hat{K}, \hat{N}],[\hat{K},[\hat{K}, \hat{N}]]] } & =16 \hat{K} .
\end{aligned}
$$

Thus the operators $\hat{K}, \hat{N}, c_{1} c_{2}-c_{2}^{\dagger} c_{1}^{\dagger}, I$ form a basis of the four dimensional Lie algebra which is not semisimple owing to $I$. The matrix representation is

$$
\hat{K}=\left(\begin{array}{cccc}
0 & 0 & 0 & 1 \\
0 & 0 & 0 & 0 \\
0 & 0 & 0 & 0 \\
1 & 0 & 0 & 0
\end{array}\right), \quad \hat{N}=\left(\begin{array}{cccc}
2 & 0 & 0 & 0 \\
0 & 1 & 0 & 0 \\
0 & 0 & 1 & 0 \\
0 & 0 & 0 & 0
\end{array}\right), \quad c_{1} c_{2}-c_{2}^{\dagger} c_{1}^{\dagger}=\left(\begin{array}{cccc}
0 & 0 & 0 & -1 \\
0 & 0 & 0 & 0 \\
0 & 0 & 0 & 0 \\
1 & 0 & 0 & 0
\end{array}\right) \text {. }
$$

For $n=3$ we have the commutators

$$
\begin{aligned}
{[\hat{K}, \hat{N}] } & =3\left(c_{1} c_{2} c_{3}-c_{3}^{\dagger} c_{2}^{\dagger} c_{1}^{\dagger}\right) \\
{[\hat{K},[\hat{K}, \hat{N}]] } & =6\left[c_{3}^{\dagger} c_{2}^{\dagger} c_{1}^{\dagger}, c_{1} c_{2} c_{3}\right] \\
{[\hat{N},[\hat{K}, \hat{N}]] } & =-9 \hat{K} \\
{[\hat{K},[\hat{K},[\hat{K}, \hat{N}]]] } & =4[\hat{K}, \hat{N}] \\
{[\hat{N},[\hat{K},[\hat{K}, \hat{N}]]] } & =0 \\
{[[\hat{K}, \hat{N}],[\hat{K},[\hat{K}, \hat{N}]]] } & =36 \hat{K} .
\end{aligned}
$$


Thus we have a four dimensional Lie algebra given by the operators $\hat{K}, \hat{N},[\hat{K}, \hat{N}]$, $[\hat{K},[\hat{K}, \hat{N}]]$. The Lie algebra is not semisimple.

For general $n$ we have

$$
\begin{aligned}
{[\hat{K}, \hat{N}] } & =n\left(c_{1} \cdots c_{n}-c_{n}^{\dagger} \cdots c_{1}^{\dagger}\right) \\
{[\hat{K},[\hat{K}, \hat{N}]] } & =2 n\left[c_{n}^{\dagger} \cdots c_{1}^{\dagger}, c_{1} \cdots c_{n}\right] \\
{[\hat{N},[\hat{K}, \hat{N}]] } & =-n^{2} \hat{K} \\
{[\hat{K},[\hat{K},[\hat{K}, \hat{N}]]] } & =4[\hat{K}, \hat{N}] \\
{[\hat{N},[\hat{K},[\hat{K}, \hat{N}]]] } & =0 \\
{[[\hat{K}, \hat{N}],[\hat{K},[\hat{K}, \hat{N}]]] } & =4 n^{2} \hat{K} .
\end{aligned}
$$

Thus we find that the four operators $\hat{K}, \hat{N},[\hat{K}, \hat{N}],[\hat{K},[\hat{K}, \hat{N}]]$ provide a basis of a four dimensional Lie algebra which is not semisimple.

\section{Entanglement}

An $n$-tangle [14, 9] can be defined for the finite dimensional Hilbert space $\mathcal{H}=\mathbb{C}^{2^{n}}$, with $n=3$ or $n$ even. Consider the finite-dimensional Hilbert space $\mathcal{H}=\mathbb{C}^{2^{n}}$ and the normalized states

$$
|\psi\rangle=\sum_{j_{1}, j_{2}, \ldots, j_{n}=0}^{1} c_{j_{1}, j_{2}, \ldots, j_{n}}\left|j_{1}\right\rangle \otimes\left|j_{2}\right\rangle \otimes \cdots \otimes\left|j_{n}\right\rangle
$$

in this Hilbert space. Here $|0\rangle,|1\rangle$ denotes the standard basis. Let $\epsilon_{j k}(j, k=0,1)$ be defined by $\epsilon_{00}=\epsilon_{11}=0, \epsilon_{01}=1, \epsilon_{10}=-1$. Let $n$ be even or $n=3$. Then an $n$-tangle can be introduced by

$$
\begin{aligned}
\tau_{1 \ldots n}= & 2 \mid \sum_{\substack{\alpha_{1}, \ldots, \alpha_{n}=0 \\
\delta_{1}, \ldots, \dot{\delta}_{n}=0}}^{1} c_{\alpha_{1} \ldots \alpha_{n}} c_{\beta_{1} \ldots \beta_{n}} c_{\gamma_{1} \ldots \gamma_{n}} c_{\delta_{1} \ldots \delta_{n}} \\
& \times \epsilon_{\alpha_{1} \beta_{1}} \epsilon_{\alpha_{2} \beta_{2}} \cdots \epsilon_{\alpha_{n-1} \beta_{n-1}} \epsilon_{\gamma_{1} \delta_{1}} \epsilon_{\gamma_{2} \delta_{2}} \cdots \epsilon_{\gamma_{n-1} \delta_{n-1}} \epsilon_{\alpha_{n} \gamma_{n}} \epsilon_{\beta_{n} \delta_{n}} \mid
\end{aligned}
$$

This includes the definition for the 3-tangle with $n=3$.

Consider now the eigenvectors of the Hamilton operator $\hat{K}$ with $n \geq 2$. Then the eigenvectors belonging to -1 and +1 are fully entangled and include part of the Bell basis. The eigenspace belonging to the eigenvalue 0 consists of both entangled and unentangled vectors. 


\section{Conclusion}

We have studied a Fermi Hamilton operator. If the eigenvalues are degenerate then by linear combinations we can construct entangled states from unentangled states. A computer algebra program written in SymbolicC $++[15]$ for the manipulation of the Fermi operators is available from the authors.

The model described above has a straightforward extension to the Fermi Hamilton operator with spin

$\hat{K}=\frac{\hat{H}}{\hbar \omega}=c_{n \uparrow}^{\dagger} c_{n-1 \uparrow}^{\dagger} \cdots c_{2 \uparrow}^{\dagger} c_{1 \uparrow}^{\dagger}+c_{n \downarrow}^{\dagger} c_{n-1 \downarrow}^{\dagger} \cdots c_{2 \downarrow}^{\dagger} c_{1 \downarrow}^{\dagger}+c_{1 \uparrow} c_{2 \uparrow} \cdots c_{n-1 \uparrow} c_{n \uparrow}+c_{1 \downarrow} c_{2 \downarrow} \cdots c_{n-1 \downarrow} c_{n \downarrow}$

with the number operator $\hat{N}$ and spin operator $\hat{S}_{z}$ given by

$$
\hat{N}=\sum_{j=1}^{n}\left(c_{j \uparrow}^{\dagger} c_{j \uparrow}+c_{j \downarrow}^{\dagger} c_{j \downarrow}\right), \quad \hat{S}_{z}=\frac{1}{2}\left(\sum_{j=1}^{n}\left(c_{j \uparrow}^{\dagger} c_{j \uparrow}-c_{j \downarrow}^{\dagger} c_{j \downarrow}\right)\right)
$$

where $[\hat{K}, \hat{N}] \neq 0,\left[\hat{K}, \hat{S}_{z}\right] \neq 0,\left[\hat{N}, \hat{S}_{z}\right]=0$. Here the matrix representation is given by [6, 7, 8, 9]

$$
\begin{gathered}
k \text {-th place } \\
c_{k \uparrow}^{\dagger}=\underbrace{\sigma_{3} \otimes \cdots \otimes \sigma_{3} \otimes\left(\frac{1}{2} \sigma_{+}\right) \otimes I_{2} \otimes \cdots \otimes I_{2}}_{2 n \text { times }} \\
c_{k \downarrow}^{\dagger}=\sigma_{3} \otimes \cdots \otimes \sigma_{3} \otimes\left(\frac{1}{2} \sigma_{+}\right) \otimes I_{2} \otimes \cdots \otimes I_{2} \\
(k+n) \text {-th place }
\end{gathered}
$$

where $k=1, \ldots, n$. Whereas the Fermi system discussed above provides a three energy level system for $n=2$ with eigenvalues $1,0,-1$ including the spin provides five energy levels with eigenvalues $2,1,0,-1,-2$ for $n=2$.

The model discussed above can easily be extended to Majorana fermions on a lattice [16, 17, 18, 19]. Given a set of $n$ (spin-less) Fermi creation and annihilations operators $c_{j}^{\dagger}, c_{j}(j=1, \ldots, n)$ we can define the set of $2 n$ (real) Majorana fermion operators on a lattice $\gamma_{j 1}, \gamma_{j 2}(j=1, \ldots, n)$ as

$$
c_{j}=\frac{1}{2}\left(\gamma_{j 1}+i \gamma_{j 2}\right), \quad c_{j}^{\dagger}=\frac{1}{2}\left(\gamma_{j 1}-i \gamma_{j 2}\right)
$$


where $\gamma_{j 1}^{*}=\gamma_{j 1}, \gamma_{j 2}^{*}=\gamma_{j 2}$ and $\gamma_{j 1}^{2}=\gamma_{j 2}^{2}=I$. It follows that

$$
\gamma_{j 1}=c_{j}^{\dagger}+c_{j}, \quad \gamma_{j 2}=i\left(c_{j}^{\dagger}-c_{j}\right)
$$

with

$$
\left[\gamma_{j 1}, \gamma_{j 2}\right]_{+}=0, \quad\left[\gamma_{j 1}, \gamma_{j 1}\right]_{+}=2 I, \quad\left[\gamma_{j 2}, \gamma_{j 2}\right]_{+}=2 I
$$

and $\left[\gamma_{j \ell}, \gamma_{k \ell^{\prime}}\right]_{+}=0$ for $j \neq k$. Another extension of the Hamilton operator would be to consider

$$
\hat{H}=J\left(b^{\dagger} \otimes c_{n}^{\dagger} c_{n-1}^{\dagger} \cdots c_{2}^{\dagger} c_{1}^{\dagger}+b \otimes c_{1} c_{2} \cdots c_{n-1} c_{n}\right)
$$

where $b^{\dagger}, b$ are Bose creation and annihilation operators with the commutation relation $\left[b, b^{\dagger}\right]=I$.

\section{Acknowledgment}

The authors are supported by the National Research Foundation (NRF), South Africa. This work is based upon research supported by the National Research Foundation. Any opinion, findings and conclusions or recommendations expressed in this material are those of the author(s) and therefore the NRF do not accept any liability in regard thereto.

\section{References}

[1] F. A. Berezin, The Method of Second Quantization, Academic Press (1966)

[2] A. L. Fetter and J. D. Walecka, Quantum Theory of Many-Particle Systems, Dover (1971)

[3] A. Auerbach, Interacting Electrons and Quantum Magnetism, SpringerVerlag, New York (1994)

[4] J. Smit, Introduction to Quantum Fields on a Lattice, Cambridge University Press, Cambridge (2002)

[5] W.-H. Steeb, “A Note on Wick's Theorem", Lett. Math. Phys. 1, 135-139 (1976)

[6] W.-H. Steeb, Problems and Solutions in Theoretical and Mathematical Physics, Third edition, Volume II: Advanced Level, World Scientific, Singapore (2009) 
[7] W.-H. Steeb and Y. Hardy, Matrix Calculus and Kronecker Product: A Practical Approach to Linear and Multilinear Algebra, 2nd edition, World Scientific, Singapore (2011)

[8] W.-H. Steeb and Y. Hardy, Problems and Solutions in Quantum Computing and Quantum Information, third edition, World Scientific, Singapore (2011)

[9] W.-H. Steeb and Y. Hardy, Quantum Mechanics using Computer Algebra, 2nd edition, World Scientific, Singapore (2010)

[10] M. Foss-Feig, A. J. Daley, J. K. Thompson and A. M. Rey, "Steady-state many-body entanglement of hot reactive fermions", Phys. Rev. Lett. 109, $230501(2012)$

[11] M. Headrick, A. Lawrence and M. Roberts, "Bose-Fermi duality and entanglement entropies", J. Stat. Phys. 1302, P02022 (2013)

[12] T. Brünner, E. Runge, A. Buchleitner and V. V. Franca, "Entanglement enhancement in spatially inhomogeneous many-body systems", Phys. Rev. A 87, $032311(2013)$

[13] M. Rodney, H. F. Song, S.-S. Lee, K. Le Hur and E. S. Sørensen, "Scaling of entanglement entropy across Lifshitz transitions", Phys. Rev. B 87, 115132 (2013)

[14] A. Wong and N. Christensen, "Potential multiparticle entanglement measure", Phys. Rev. A 63, 044301 (2001)

[15] Y. Hardy, Tan Kiat Shi and W.-H. Steeb, Computer Algebra with SymbolicC++, World Scientific, Singapore (2008)

[16] S. B. Bravyi and A. Yu. Kitaev, "Fermionic quantum computation", arXiv:quant-ph/0003137v2

[17] A. Yu. Kitaev and C. Laumann, "Topological phases and quantum computation", arXiv:0904.2771v1

[18] L. H. Kauffman, "Knot Logic and Topological Quantum Computing with Majorana Fermions", arXiv:quant-ph/1301.6214v1

[19] A. Jaffe and F. L. Pedrocchi, "Reflection Positivity for Majorana Fermions", arXiv:math-ph/1305.1792v1 